\title{
Constraints on the neutron skin and symmetry energy from the anti-analog giant dipole resonance in ${ }^{208} \mathrm{~Pb}$
}

\author{
Li-Gang Cao, ${ }^{1,2,3,4}$ X. Roca-Maza, ${ }^{5,6}$ G. Colò, ${ }^{5,6,3}$ and H. Sagawa ${ }^{7,8,3}$ \\ ${ }^{1}$ School of Mathematics and Physics, North China Electric Power University, Beijing 102206, China \\ ${ }^{2}$ State Key Laboratory of Theoretical Physics, Institute of Theoretical Physics, Chinese Academy of Sciences, Beijing 100190, China \\ ${ }^{3}$ Kavli Institute for Theoretical Physics China, CAS, Beijing 100190, China \\ ${ }^{4}$ Center of Theoretical Nuclear Physics, National Laboratory of Heavy Ion Accelerator of Lanzhou, Lanzhou 730000, China \\ ${ }^{5}$ Dipartimento di Fisica, Università degli Studi di Milano, via Celoria 16, 20133 Milano, Italy \\ ${ }^{6}$ Istituto Nazionale di Fisica Nucleare (INFN), Sezione di Milano, via Celoria 16, 20133 Milano, Italy \\ ${ }^{7}$ Center for Mathematics and Physics, University of Aizu, Aizu-Wakamatsu, Fukushima 965-8580, Japan \\ ${ }^{8}$ RIKEN, Nishina Center, Wako, 351-0198, Japan \\ (Received 23 April 2015; published 10 September 2015)
}

\begin{abstract}
We investigate the impact of the neutron skin thickness, $\Delta R_{n p}$, on the energy difference between the anti-analog giant dipole resonance (AGDR), $E_{\mathrm{AGDR}}$, and the isobaric analog state (IAS), $E_{\mathrm{IAS}}$, in a heavy nucleus such as ${ }^{208} \mathrm{~Pb}$. For guidance, we first develop a simple and analytic, yet physical, approach based on the droplet model that linearly connects the energy difference $E_{\mathrm{AGDR}}-E_{\mathrm{IAS}}$ with $\Delta R_{n p}$. To test this correlation on more fundamental grounds, we employ a family of systematically varied Skyrme energy density functionals where variations on the value of the symmetry energy at saturation density $J$ are explored. The calculations have been performed within the fully self-consistent Hartree-Fock (HF) plus charge-exchange random phase approximation (RPA) framework. We confirm the linear correlation within our microscopic approach and we can compare our results with available experimental data in ${ }^{208} \mathrm{~Pb}$ in order to extract a preferred value for $\Delta R_{n p}$ and, in turn, for the symmetry energy parameters. Averaging the results from two available experimental data, our analysis gives $\Delta R_{n p}=0.236 \pm 0.018 \mathrm{fm}, J=33.2 \pm 1.0 \mathrm{MeV}$, and a slope parameter of the symmetry energy at saturation $L=97.3 \pm 11.2 \mathrm{MeV}$. The errors include the experimental uncertainties and a lower-limit estimate of model uncertainties. These results are consistent with those extracted from different experimental data albeit $L$ and $\Delta R_{n p}$ are somewhat large when compared to previous estimations based on giant resonance studies. Possible hints whether model dependence can explain this difference are provided.
\end{abstract}

\section{INTRODUCTION}

Different experimental methods, either direct or indirect, have been proposed to extract the value of the neutron skin thickness in finite nuclei, that is, the difference between neutron and proton root-mean-square radii,

$$
\Delta R_{n p} \equiv\left\langle r^{2}\right\rangle_{n}^{1 / 2}-\left\langle r^{2}\right\rangle_{p}^{1 / 2} .
$$

The neutron skin thickness is an observable that has kept much attention from both experimental and theoretical viewpoints. This is because it is one of the most promising observables in nuclear structure to constrain the density dependence of the symmetry energy around the nuclear saturation density [1-7]. The symmetry energy plays an important role in understanding the mechanisms of different phenomena in nuclear physics and nuclear astrophysics [8-28]: it directly affects the properties of exotic nuclei, the dynamics of heavy-ion collisions, the structure of neutron stars, and the simulations of core-collapse supernova.

The Lead Radius Experiment (PREX) at the Jefferson Laboratory has provided the first model-independent evidence on the existence of a neutron-rich skin in ${ }^{208} \mathrm{~Pb}$ [29]. Relying on the fact that the weak charge of the neutron is much larger than the corresponding proton one, PREX used parityviolating electron scattering to probe the neutron distribution of ${ }^{208} \mathrm{~Pb}$. To foster this field, more experiments have been already approved with both the aim of improving the reached accuracy in ${ }^{208} \mathrm{~Pb}$ and exploring other mass regions. On the other side, neutron densities have been traditionally probed mostly by nucleon or $\alpha$ scattering, for example, by using proton elastic scattering on $\mathrm{Sn}$ and $\mathrm{Pb}$ isotopes [30] or by measuring photons emitted during the decay of antiproton states $[31,32]$. One can also obtain information on the neutron skin thickness from giant resonance properties, such as the excitation energy of the isovector giant dipole resonance (IVGDR), the total electric dipole polarizability $\left(\alpha_{D}\right)$, the excitation energy of the isovector giant quadrupole resonance (IVGQR), or, yet with more warnings, from the energy and strength of the pygmy dipole resonance (PDR) in neutron-rich nuclei [33-45]. Last but not least, the total strength of the charge-exchange spin-dipole resonances (SDR) can be related to the neutron skin in a very transparent way [7,46-51]. It is important to mention, however, that all hadronic probes require model assumptions to deal with the strong force introducing possible systematic uncertainties.

Recently, the authors of Refs. [52-54] have proposed a new method to extract the neutron skin thickness based on the measurement of the excitation energy of the anti-analog giant dipole resonance (AGDR), that can be observed in the charge-exchange $(p, n)$ reaction. The AGDR was first studied experimentally in Ref. [55]. Already in Ref. [56], the authors had pointed out that the excitation energy of the AGDR is 
sensitive to the neutron skin thickness. More recently, the energy difference between the AGDR and the isobaric analog state (IAS), $E_{\mathrm{AGDR}}-E_{\mathrm{IAS}}$, in ${ }^{208} \mathrm{~Pb}$ has been obtained by measuring the direct $\gamma$ decay between these states [57].

In this paper, we shall analyze the relationship of the neutron skin thickness and the energy difference $E_{\mathrm{AGDR}}-E_{\mathrm{IAS}}$, by using a fully self-consistent Hartree-Fock (HF) plus chargeexchange random phase approximation (RPA) framework with a family of Skyrme energy density functionals. We try to understand also the qualitative features of such a relationship through a simple yet physical and transparent model. Our approach, as compared to Ref. [56], incorporates specific effects of the Skyrme functionals such as the effective mass and the isovector enhancement factor (cf. Sec. III). Then, by comparing the theoretical and experimental results for $E_{\mathrm{AGDR}}-E_{\mathrm{IAS}}$, we extract the neutron skin thickness in ${ }^{208} \mathrm{~Pb}$. This allows us, in turn, to estimate the compatible values for the symmetry energy $J$ and its slope parameter $L$ (at nuclear matter saturation density). The extracted values of $J$ and $L$ are eventually compared to the results obtained by another analysis on different observables.

The outline of the paper is as follows. In Sec. II the theoretical model is briefly presented: we focus, in particularly on the charge-exchange random phase approximation (RPA) based on the use of nonrelativistic Skyrme energy density functionals (EDFs). In Sec. III, we derive our analytic model to explain the relationship between the energy difference $E_{\mathrm{AGDR}}-E_{\mathrm{IAS}}$ of AGDR and IAS, and the neutron skin thickness. A detailed quantitative analysis of such a correlation is performed by employing a family of so-called SAMi-J Skyrme functionals, in Sec. IV. In Sec. V we summarize the results and draw our conclusions.

\section{MICROSCOPIC MODEL: CHARGE-EXCHANGE RPA}

The calculations are done within the framework of the Skyrme HF [58] plus charge-exchange RPA. We adopt the standard form of Skyrme interactions with the notations of Ref. [59]. Two nucleons characterized by the space, spin, and isospin variables $\boldsymbol{r}_{i}, \boldsymbol{\sigma}_{i}$, and $\boldsymbol{\tau}_{i}$ interact through a zero-range, velocity-dependent, and density-dependent force that reads

$$
\begin{aligned}
V\left(\boldsymbol{r}_{1}, \boldsymbol{r}_{2}\right)= & t_{0}\left(1+x_{0} P_{\sigma}\right) \delta(\boldsymbol{r}) \\
& +\frac{1}{2} t_{1}\left(1+x_{1} P_{\sigma}\right)\left[\boldsymbol{P}^{\prime 2} \delta(\boldsymbol{r})+\delta(\boldsymbol{r}) \boldsymbol{P}^{2}\right] \\
& +t_{2}\left(1+x_{2} P_{\sigma}\right) \boldsymbol{P}^{\prime} \cdot \delta(\boldsymbol{r}) \boldsymbol{P} \\
& +\frac{1}{6} t_{3}\left(1+x_{3} P_{\sigma}\right) \rho^{\alpha}(\boldsymbol{R}) \delta(\boldsymbol{r}) \\
& +i W_{0}\left(\sigma_{1}+\sigma_{2}\right) \cdot\left[\boldsymbol{P}^{\prime} \times \delta(\mathbf{r}) \boldsymbol{P}\right],
\end{aligned}
$$

where $\boldsymbol{r}=\boldsymbol{r}_{1}-\boldsymbol{r}_{2}, \boldsymbol{R}=\frac{1}{2}\left(\boldsymbol{r}_{1}+\boldsymbol{r}_{2}\right), \boldsymbol{P}=\frac{1}{2 i}\left(\nabla_{1}-\nabla_{2}\right), \boldsymbol{P}^{\prime}$ is the Hermitian conjugate of $\boldsymbol{P}$ (acting on the left), $P_{\sigma}=\frac{1}{2}(1+$ $\left.\sigma_{1} \cdot \sigma_{2}\right)$ is the spin-exchange operator, and $\rho=\rho_{n}+\rho_{p}$ is the total nucleon density. Within the standard formalism, the total binding energy of a nucleus can be expressed as the integral of the Skyrme density functional [59], which includes the kineticenergy term $\mathcal{K}$, a zero-range term $\mathcal{H}_{0}$, the density-dependent term $\mathcal{H}_{3}$, an effective-mass term $\mathcal{H}_{\text {eff }}$, a momentum dependent term (that mimics finite-range effects) $\mathcal{H}_{\text {fin }}$, a spin-orbit term $\mathcal{H}_{\text {so }}$, a spin-gradient term $\mathcal{H}_{\text {sg }}$, and a Coulomb term $\mathcal{H}_{\text {Coul }}$.
Here, we will briefly summarize the formulas for the charge-exchange RPA calculations. The well-known RPA method [60,61] in matrix form is given by

$$
\left(\begin{array}{cc}
A & B \\
B^{*} & A^{*}
\end{array}\right)\left(\begin{array}{l}
X^{v} \\
Y^{v}
\end{array}\right)=E_{\nu}\left(\begin{array}{cc}
1 & 0 \\
0 & -1
\end{array}\right)\left(\begin{array}{l}
X^{v} \\
Y^{v}
\end{array}\right),
$$

where $E_{v}$ is the energy of the $v$ th charge-exchange RPA state and $X^{v}, Y^{v}$ are the corresponding forward and backward amplitudes, respectively. The matrix elements $A$ and $B$ are expressed as

$$
\begin{aligned}
& A_{m i, n j}=\left(\epsilon_{m}-\epsilon_{n}\right) \delta_{m n} \delta_{i j}+\left\langle m j\left|V_{\text {res }}\right| i n\right\rangle, \\
& B_{m i, n j}=\left\langle m n\left|V_{\text {res }}\right| i j\right\rangle,
\end{aligned}
$$

where the subindexes $i, j$ refer to occupied states, $m, n$ to unoccupied states, $V_{\text {res }}$ is the residual interaction, and $\epsilon$ the single-particle state energy. The particle-hole (p-h) matrix elements are obtained from the Skyrme energy density functional including all the terms (the Coulomb term $\mathcal{H}_{\text {Coul }}$ is not active in this case).

Our charge-exchange RPA had been first introduced and illustrated in Ref. [62], to which we refer the reader for details. Some details that are common between charge-exchange and normal RPA can be found in Ref. [63], where HF-RPA for non charge-exchange models is fully discussed.

We will use the following operator for the AGDR excitation:

$$
\hat{O}_{ \pm}=\sum_{i} r_{i} Y_{1 m}\left(\hat{r}_{i}\right) t_{ \pm}^{(i)}
$$

which corresponds to the $\Delta J=1, \Delta L=1, \Delta S=0, J^{\pi}=1^{-}$ resonance. We will also calculate the IAS. The IAS excitation operator reads

$$
\hat{O}_{I A S}=\sum_{i} t_{ \pm}^{(i)}
$$

and corresponds the $\Delta J=0, \Delta L=0, \Delta S=0, J^{\pi}=0^{+}$ states.

In Fig. 1, we illustrate the relevant states of a target and its daughter nucleus, namely the ground state, the giant dipole resonance, and the giant $M 1$ state of a target nucleus. $T_{0} \neq 0$ labels the ground-state isospin of the target nucleus, that is, $(N-Z) / 2$. The corresponding resonance states in the daughter nucleus reached by the $(p, n)$ charge-exchange reaction are also displayed, namely the IAS (isospin $=T_{0}$ ), and the anti-analog (isospin $=T_{0}-1$ ) states: Gamow-Teller resonance (GTR), SDR, and AGDR. As shown in the figure, the AGDR corresponds to $\Delta J^{\pi}=1^{-}, \Delta L=1$, and $\Delta S=0$ excitation, and represents the anti-analog giant dipole resonance because it is the $T_{0}-1$ component of the charge-exchange of the isovector giant dipole resonance (IVGDR).

\section{GUIDELINE FROM A SIMPLE ANALYTIC MODEL}

In this section we will develop a simple, yet physically sound, model for the excitation energies of the IVGDR, the IAS, and the AGDR. This effort will allow us to gain a deeper insight into the relevant "macroscopic" physics of our problem, namely the relationship between the neutron skin thickness and the energy difference of the AGDR and the IAS. 


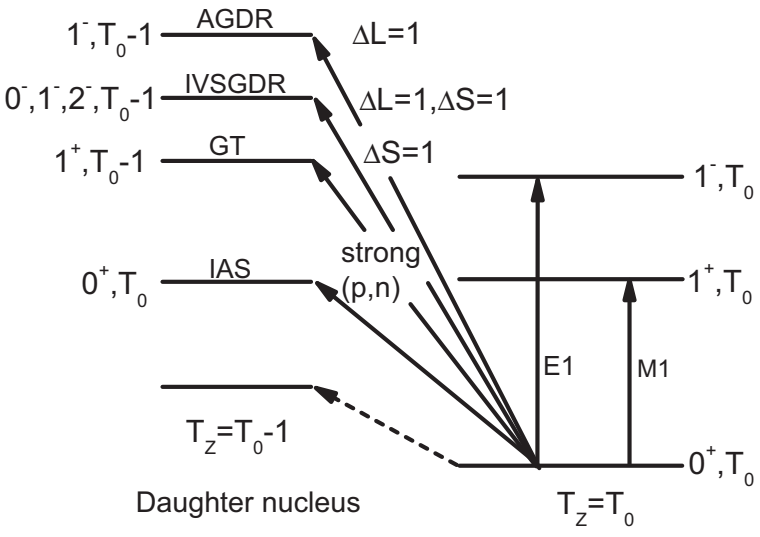

Target nucleus

FIG. 1. Various states related to the target and daughter nucleus, including the ground state, the IVGDR and $M 1$ excitations of the target nucleus (isospin $=T_{0}$ ), and the IAS (isospin $=T_{0}$ ), anti-analog states (isospin $\left.=T_{0}-1\right)$ in the daughter nucleus excited in a $(p, n)$ reaction.

We start from the RPA dispersion relation for a separable interaction of the type $\kappa \hat{O}^{\dagger} \hat{O}$, that is,

$$
\sum_{p h} \frac{|\langle p|\hat{O}| h\rangle|^{2}}{\varepsilon_{p h}-E}+\frac{|\langle p|\hat{O}| h\rangle|^{2}}{\varepsilon_{p h}+E}=-\frac{1}{\kappa} .
$$

In this equation, $\varepsilon_{p h}$ is the unperturbed p-h excitation energy; if we assume that there is only one unperturbed configuration at energy $\varepsilon_{p h}=\varepsilon_{0}$ that exhausts the whole unperturbed strength $S_{0}$, then the equation becomes

$$
\frac{S_{0}}{\varepsilon_{0}-E}+\frac{S_{0}}{\varepsilon_{0}+E}=-\frac{1}{\kappa}
$$

and, therefore,

$$
E^{2}=\varepsilon_{0}^{2}+2 \kappa m_{0},
$$

where $m_{0}=\varepsilon_{0} S_{0}$ is the energy weighted sum rule (EWSR).

For each mode, there will be a different coupling constant; however, the isospin invariance dictates that the coupling constants of the AGDR and the non charge-exchange IVGDR are the same. We start by considering the well-known IVGDR case: $\hat{O}=\sum_{i} r_{i} Y_{10}\left(\hat{r}_{i}\right) \tau_{z}^{(i)}$-note that for the macroscopic model we use the components of $\tau$ instead of $t$.

We will use the Bohr-Mottelson quantal harmonic oscillator (QHO) model [64]; in this case, the coupling constant $\kappa$ for a dipole excitation is determined by the self-consistent condition between the vibrating potential and density:

$$
\begin{aligned}
\kappa_{\lambda=1}^{\tau=0} & =-\frac{4 \pi}{3 A} m \omega_{0}^{2}, \\
\kappa_{\lambda=1}^{\tau=1} & =\frac{\pi V_{1}}{A\left\langle r^{2}\right\rangle}
\end{aligned}
$$

for an isoscalar $(\tau=0)$ or isovector $(\tau=1)$ dipole $(\lambda=1)$ excitations, respectively. $\hbar \omega_{0}$ is the major shell gap, $\approx 41$ $A^{-1 / 3} \mathrm{MeV}, V_{1}$ is the strength of Lane potential $U=V_{1} \frac{N-Z}{2 A}$, and $\left\langle r^{2}\right\rangle$ is the mean square radius. The classical EWSR of the isovector dipole mode is given by

$$
m_{0}(\tau=1, \lambda=1)=\frac{3}{4 \pi} \frac{\hbar^{2}}{2 m} A .
$$

It has to be noted that the energy $E_{\lambda=1}^{\tau=0}$ of the isoscalar dipole mode (viz., the spurious center-of-mass mode) turns out to be at zero energy, as it should be. This can be checked from Eqs. (10) and (13). Moreover, the energy of the isovector giant dipole resonance, $E_{\lambda=1}^{\tau=1} \equiv E_{\mathrm{IVGDR}}$, becomes

$$
E_{\mathrm{IVGDR}}^{2}=\varepsilon_{0}^{2}\left(1+\frac{3 \hbar^{2} V_{1}}{4\left\langle r^{2}\right\rangle m \varepsilon_{0}^{2}}\right),
$$

and it is well known that by using standard values for the unperturbed energy $\left(\varepsilon_{0}=\hbar \omega_{0}=41 / A^{1 / 3} \mathrm{MeV}\right)$ as well as for the radius $\left(\left\langle r^{2}\right\rangle=3 R_{0}^{2} / 5=3(1.2)^{2} A^{2 / 3} / 5 \mathrm{fm}^{2}\right)$, together with $V_{1}=130 \mathrm{MeV}$, the excitation energy provided by Eq. (14) is $E_{\lambda=1}^{\tau=1} \approx 80 / A^{1 / 3} \mathrm{MeV}$, in good agreement with the empirical systematics for the IVGDR in the mass region $A>40$.

There are two important differences between the interaction assumed in the Bohr-Mottelson model and the Skyrme interaction. In the former case, the effective mass is taken to be $m^{*} / m=1$, while for Skyrme interactions this value depends on the chosen set, being in uniform matter as well as in the interior of nuclei close to the empirical value, $\mathrm{m}^{*} / \mathrm{m} \approx 0.7$. The effective mass changes the unperturbed energy to be $\varepsilon=\varepsilon_{0} \sqrt{\frac{m}{m^{*}}}$. Moreover, in the case of momentum-dependent interactions such as the Skyrme forces (or other nonlocal forces), the classical EWSR should be multiplied by $1+\alpha$ where $\alpha$ is the so-called enhancement factor. For the dipole case, $\alpha$ is typically around $\approx 0.2$ [65]. Consequently, Eq. (10) should turn into

$$
E^{2}=\frac{m}{m^{*}} \varepsilon_{0}^{2}+2 \kappa^{\prime} m_{0}(1+\alpha) .
$$

It is a simple exercise to show that Eq. (15) reproduces the experimental IVGDR systematics as well as Eq. (10) if the coupling constant $\kappa^{\prime}$ is reduced with respect to $\kappa$, that is, $\kappa^{\prime} \approx 0.7 \kappa$. By inspecting Eq. (12) we can also conclude that this implies a quenched value for the strength of the Lane potential $V_{1}^{\prime}$ with $V_{1}^{\prime} \approx 0.7 V_{1}$ (this value is of course indicative, in keeping with the rather crude approximations of this analytic model).

In the case of charge-exchange excitations of nuclei having a neutron excess, like ${ }^{208} \mathrm{~Pb}$, the Tamm-Dancoff approximation (TDA) is known to provide results that are quite similar to those from the RPA. This is because the coupling between the $\tau_{-}$excitations and $\tau_{+}$excitations is small due to their quite large energy difference. We simplify thus the RPA dispersion relation to be the TDA one,

$$
\sum_{p h} \frac{|\langle p|\hat{O}| h\rangle|^{2}}{\varepsilon_{p h}-E}=-\frac{1}{\kappa} .
$$

As we have done already, we assume that there is only one unperturbed configuration at energy $\varepsilon_{p h}=\varepsilon_{0}$ exhausting the whole unperturbed strength $S_{0}$, so that

$$
\frac{S_{0}}{\varepsilon_{0}-E}=-\frac{1}{\kappa} \text {. }
$$


The solution of this simplified TDA equation is

$$
\begin{aligned}
E & =\varepsilon_{0}+\kappa S_{0} \\
& =\varepsilon_{0}+\kappa \frac{m_{0}}{\varepsilon_{0}} .
\end{aligned}
$$

Let us consider the IAS first. It is well known that, to a first approximation, its excitation energy is associated with the Coulomb energy shift $\Delta E_{C}$ between the parent and the daughter nuclei. In our TDA model we can write the unperturbed $p-h$ energy as $\varepsilon_{0}=-U+\Delta E_{C}$, where $U$ is, as above, the Lane potential. The non energy weighted sum rule (NEWSR) obtained by using the operator $\sum_{i} \tau_{-}(i)$ is $2(N-Z)$. Therefore,

$$
E_{\mathrm{IAS}}=-U+\Delta E_{C}+\kappa_{\lambda=0}^{\tau=1} 2(N-Z)
$$

and if

$$
\kappa=\frac{V_{1}}{4 A}
$$

the IAS energy coincides with $\Delta E_{C}$.

Let us finally move to the subject of main interest for us, namely the AGDR. Our goal is to have a transparent interpretation of the results obtained with the microscopic Skyrme model.

The AGDR has $\Delta L=1, \Delta S=0$, where the corresponding operator is

$$
\sum_{i} r_{i} Y_{10} \tau_{-}(i)
$$

The NEWSR reads

$$
S_{0}\left(\tau_{-}, \lambda=1\right)-S_{0}\left(\tau_{+}, \lambda=1\right)=\frac{(N-Z)}{2 \pi}\left\langle r^{2}\right\rangle_{n e},
$$

where

$$
\left\langle r^{2}\right\rangle_{n e} \equiv \frac{N\left\langle r^{2}\right\rangle_{n}-Z\left\langle r^{2}\right\rangle_{p}}{N-Z}
$$

In this sum rule the $\tau_{-}$contribution is largely dominant in nuclei with neutron excess like ${ }^{208} \mathrm{~Pb}$; the same dominance holds for the EWSR, that can be written as

$$
m_{0}\left(\tau_{-}, \lambda=1\right)-m_{0}\left(\tau_{+}, \lambda=1\right)=\frac{3}{2 \pi} \frac{\hbar^{2} A}{2 m}(1+\alpha+\beta) .
$$

$\alpha$ is the same as in the IVGDR case that we have discussed above, whereas the definition of $\beta$ can be found, in the case of a Skyrme interaction, in Ref. [66].

Within the framework of our approximation, the AGDR unperturbed energy can be written as $\varepsilon-U+\Delta E_{C}$; consequently, its TDA energy from the simplified equation (18) reads

$$
\begin{aligned}
E_{\mathrm{AGDR}} & =\varepsilon-U+\Delta E_{C}+\frac{V_{1}^{\prime}}{2} \frac{\left(N\left\langle r^{2}\right\rangle_{n}-Z\left\langle r^{2}\right\rangle_{p}\right)}{A\left\langle r^{2}\right\rangle}, \\
& =\varepsilon-U+\Delta E_{C}+\frac{V_{1}^{\prime}}{\left\langle r^{2}\right\rangle} \frac{\frac{3}{2} \frac{\hbar^{2}}{2 m}(1+\alpha+\beta)}{\varepsilon-U+\Delta E_{C}}
\end{aligned}
$$

We are supposed to use the same coupling constant that has been already used in the case of the IVGDR; according to
TABLE I. Excitation energies and NEWSRs $\left(m_{0}\right)$ of the AGDR and IVGDR for the family of SAMi-J interactions are displayed, together with the excitation energies of the IAS, the enhancement factors $\gamma=\beta /(1+\alpha)$, and the predictions of the macroscopic model $E_{\mathrm{AGDR}}^{\mathrm{mac}}$ estimated from Eq. (34). To evaluate $E_{\mathrm{AGDR}}^{\mathrm{mac}}$, we take $\varepsilon=41 A^{-1 / 3} \sqrt{m / m^{*}} \mathrm{MeV}, \Delta E_{C}=2(3 / 5)^{3 / 2} e^{2} Z /\left\langle r^{2}\right\rangle^{1 / 2}$, and the energies of the current table. See the text for more details.

\begin{tabular}{lcccccccc}
\hline \hline Force & $\begin{array}{c}E_{\mathrm{AGDR}} \\
{[\mathrm{MeV}]}\end{array}$ & $\begin{array}{c}m_{0}^{\text {AGDR }} \\
{\left[\mathrm{fm}^{2}\right]}\end{array}$ & $\begin{array}{c}E_{\mathrm{IVGDR}} \\
{[\mathrm{MeV}]}\end{array}$ & $\begin{array}{c}m_{0}^{\text {IVGDR }} \\
{\left[\mathrm{fm}^{2}\right]}\end{array}$ & $\begin{array}{c}E_{\mathrm{IAS}} \\
{[\mathrm{MeV}]}\end{array}$ & $\gamma$ & $\begin{array}{c}E_{\mathrm{AGDR}}^{\mathrm{mac}} \\
{[\mathrm{MeV}]}\end{array}$ \\
\hline SAMi-J27 & 28.20 & 11153.99 & 13.99 & 4073.4 & 18.75 & 0.369 & 25.51 \\
SAMi-J28 & 27.94 & 11119.60 & 13.91 & 4071.0 & 18.60 & 0.366 & 25.25 \\
SAMi-J29 & 27.64 & 11079.18 & 13.74 & 4067.9 & 18.43 & 0.362 & 24.87 \\
SAMi-J30 & 27.37 & 11055.00 & 13.58 & 4065.4 & 18.29 & 0.360 & 24.51 \\
SAMi-J31 & 27.16 & 11047.33 & 13.42 & 4063.5 & 18.13 & 0.359 & 24.18 \\
SAMi-J32 & 26.94 & 11055.63 & 13.27 & 4062.6 & 18.00 & 0.361 & 23.89 \\
SAMi-J33 & 26.73 & 11078.24 & 13.13 & 4061.6 & 17.89 & 0.364 & 23.62 \\
SAMi-J34 & 26.54 & 11113.27 & 13.00 & 4061.3 & 17.81 & 0.368 & 23.40 \\
SAMi-J35 & 26.39 & 11158.82 & 12.88 & 4061.4 & 17.74 & 0.374 & 23.21 \\
\hline \hline
\end{tabular}

our previous discussion, this will be different from the case of the Bohr-Mottelson model if used in conjunction either with an effective mass and/or with an enhancement factor as in the Skyrme case. For convenience, we shall define here $\bar{V}_{1} \equiv V_{1}^{\prime}(1+\alpha)$-note that $\bar{V}_{1}=V_{1}$ if $m^{*} / m=1$ and $\bar{V}_{1} \approx V_{1}$ even for realistic models with $m^{*} / m<1$. One can also notice that a simplification of Eq. (25) comes from the fact that for a heavy nucleus such as ${ }^{208} \mathrm{~Pb}$, replacing $\varepsilon-U+\Delta E_{C}$ with $\Delta E_{C}$ will produce an error of only a few $\%$. Specifically, if we assume $m * / m \approx 0.7$ and $V_{1} \approx$ $130 \mathrm{MeV}$ as previously done, $\varepsilon-U=41 A^{-1 / 3} \sqrt{\mathrm{m} / \mathrm{m}^{*}}-$ $V_{1}^{\prime}(N-Z) / 2 A \approx 1.3 \mathrm{MeV}$ which corresponds to about $7 \%$ when compared to $\Delta E_{C}$. We use this simplification to write the energy difference between the AGDR and the IAS:

$$
\begin{aligned}
E_{\mathrm{AGDR}}-E_{\mathrm{IAS}} & =\frac{\bar{V}_{1}}{2(1+\alpha)} \frac{\left(N\left\langle r^{2}\right\rangle_{n}-Z\left\langle r^{2}\right\rangle_{p}\right)}{A\left\langle r^{2}\right\rangle}, \\
& =\frac{\bar{V}_{1}}{\left\langle r^{2}\right\rangle} \frac{\frac{3}{2} \frac{\hbar^{2}}{2 m}(1+\alpha+\beta)}{\Delta E_{C}(1+\alpha)} .
\end{aligned}
$$

It is convenient to define the quantity $\gamma \equiv \beta /(1+\alpha)$ since it is almost constant if we consider the interactions employed in the current study (see Table I). Finally, approximating the IAS energy as the Coulomb shift energy between parent and daughter nuclei by $\Delta E_{C}=2(3 / 5)^{3 / 2} e^{2} Z /\left\langle r^{2}\right\rangle^{1 / 2}$, we may write

$$
\begin{aligned}
E_{\mathrm{AGDR}}-E_{\mathrm{IAS}} & =\frac{\bar{V}_{1}(1+\gamma)}{\Delta E_{C}} \frac{3}{2} \frac{\hbar^{2} c^{2}}{2 m c^{2}\left\langle r^{2}\right\rangle} \\
& \approx \frac{5}{8} \sqrt{\frac{5}{3}} \frac{\bar{V}_{1}(1+\gamma)}{\alpha_{\mathrm{H}} Z} \frac{\hbar c}{m c^{2}\left\langle r^{2}\right\rangle^{1 / 2}} .
\end{aligned}
$$

If we take $\alpha \approx 0.2, \gamma \approx 0.4$, and $V_{1} \approx 130 \mathrm{MeV}$, we find $E_{\mathrm{AGDR}}-E_{\mathrm{IAS}} \approx 9 \mathrm{MeV}$, which is in reasonable agreement with the result of our realistic calculations.

This schematic model gives us the opportunity to understand the sensitivity of $E_{\mathrm{AGDR}}-E_{\mathrm{IAS}}$ on the neutron skin thickness $\Delta R_{n p}$. In fact, as it was done in Ref. [42] to which 
we refer the reader for details, we can relate the interaction strength of the potential $\bar{V}_{1}$ with the neutron skin thickness via the droplet model (DM),

$$
\bar{V}_{1} \approx 8\left[a_{\text {sym }}(A)-\varepsilon_{F_{\infty}} / 3\right] .
$$

The DM also predicts that

$$
\begin{aligned}
J & -a_{\mathrm{sym}}(A) \\
& \approx \frac{3 J}{2\left\langle r^{2}\right\rangle^{1 / 2}} \frac{1}{I-I_{C}}\left(\Delta R_{n p}-\Delta R_{n p}^{\mathrm{surf}}+\frac{2}{7} I_{C}\left\langle r^{2}\right\rangle^{1 / 2}\right),
\end{aligned}
$$

where $I_{C}=e^{2} Z / 20 J R$ is a Coulomb correction to the total neutron excess $I=(N-Z) / A, a_{\text {sym }}(A)$ is the symmetry energy parameter of the DM, and $\Delta R_{n p}^{\text {surf }}$ is a surface correction to the neutron skin thickness due to the different neutron and proton surface diffuseness. The latter quantity has been shown to be approximately constant in ${ }^{208} \mathrm{~Pb}\left(\Delta R_{n p}^{\text {surf }} \approx 0.09 \pm\right.$ $0.01 \mathrm{fm}$ ) when calculated by a large set of energy density functionals of different kinds [67]. Since $I_{C}$ corresponds to a correction of about a $10 \%$ to $I$ in heavy neutron-rich nuclei such as ${ }^{208} \mathrm{~Pb}$, we will assume in what follows that $I-I_{C} \approx I$, and find

$$
J-a_{\text {sym }}(A) \approx \frac{3 J}{2 I} \times \frac{\Delta R_{n p}-\Delta R_{n p}^{\text {surf }}}{\left\langle r^{2}\right\rangle^{1 / 2}}+\frac{3}{7} \frac{I_{C}}{I} J,
$$

and by combining this result with Eq. (27) one finds that

$$
\begin{aligned}
E_{\mathrm{AGDR}}-E_{\mathrm{IAS}} \approx & 5 \sqrt{\frac{5}{3}} \frac{J}{I} \frac{1+\gamma}{\alpha_{\mathrm{H}} Z} \frac{\hbar c}{m\left\langle r^{2}\right\rangle^{1 / 2}}\left[\left(1-\frac{\varepsilon_{F_{\infty}}}{3 J}\right) I\right. \\
& \left.-\frac{3}{2}\left(\frac{\Delta R_{n p}-\Delta R_{n p}^{\mathrm{surf}}}{\left\langle r^{2}\right\rangle^{1 / 2}}\right)-\frac{3}{7} I_{C}\right]
\end{aligned}
$$

For a given nucleus, Eq. (31) predicts an explicit linear anticorrelation of $E_{\mathrm{AGDR}}-E_{\mathrm{IAS}}$ with $\Delta R_{n p}$. We will show in the next section that this correlation is actually displayed by the microscopic results.

We have also found very instructive to relate the different excitation energies within our macroscopic model, and check if the microscopic results follow such relationship. In doing that we have used the TDA expressions for the IVGDR, IAS, and AGDR, and after some algebra, we arrive at

$$
\begin{aligned}
E_{\mathrm{AGDR}}= & \Delta E_{C}\left(1+\frac{\varepsilon-U}{\Delta E_{C}}\right) \\
& +\left(E_{\mathrm{IVGDR}}-\varepsilon\right) 2(1+\gamma) \frac{\varepsilon}{\Delta E_{C}} \frac{1}{1+\frac{\varepsilon-U}{\Delta E_{C}}} .
\end{aligned}
$$

As previously done, in a nucleus such as ${ }^{208} \mathrm{~Pb},(\varepsilon-U) / \Delta E_{C}$ can be neglected. Therefore, within a good approximation, we can write

$$
E_{\mathrm{AGDR}}-E_{\mathrm{IAS}} \approx \frac{\varepsilon}{\Delta E_{C}}\left(E_{\mathrm{IVGDR}}-\varepsilon\right) 2(1+\gamma)
$$

or

$$
E_{\mathrm{AGDR}}-E_{\mathrm{IAS}} \approx \frac{\varepsilon}{\Delta E_{C}}\left(E_{\mathrm{IVGDR}}-\varepsilon\right) \frac{m_{0}^{\mathrm{AGDR}}}{m_{0}^{\mathrm{IVGDR}}} .
$$

We define the energy of the AGDR extracted from Eq. (34) as $E_{\text {AGDR }}^{\mathrm{mac}}$, in Table I and hereafter. For the SAMi-J family and for a fixed nucleus, this formula suggests that the energy difference $E_{\mathrm{AGDR}}-E_{\mathrm{IAS}}$ should display the same trend as shown by $E_{\text {IVGDR }}$. In fact, $\varepsilon$ depends only on the effective mass which is constant for the SAMi-J family $\left(\varepsilon \equiv 41 A^{-1 / 3} \sqrt{m / m^{*}} \mathrm{MeV}\right)$. Moreover, $\Delta E_{C}$ is expected to not vary, and $1+\gamma$ is also approximately constant (cf. Table I). The expression (34) reflects the idea that the physics encoded in the energy difference $E_{\mathrm{AGDR}}-E_{\mathrm{IAS}}$ reflects that of the IVGDR, as expected because of isospin invariance.

In Table I, we present the predictions of the SAMi-J family for the different observables under study. The reader can verify that the latter equations of this section reasonably reproduce the microscopic HF-RPA results: although there is an almost constant shift, the trend of $E_{\mathrm{AGDR}}(\mathrm{RPA})$, however, is almost perfectly reproduced by the value $E_{\mathrm{AGDR}}^{\mathrm{mac}}$ in Eq. (34). This finding gives us confidence in using the simple arguments in this section to interpret the microscopic results.

\section{RESULTS AND DISCUSSIONS}

In this section, we discuss the results obtained by employing the SAMi-J Skyrme energy density functionals to calculate the HF ground state and RPA excited states. The SAMi$\mathbf{J}$ interactions are characterized by different values of the symmetry energy at saturation density: this value varies between $27 \mathrm{MeV}$ and $35 \mathrm{MeV}$ (in steps of $1 \mathrm{MeV}$ ), and the force parameters are fitted using properties of selected nuclei while keeping at the same time the constraints on a few properties of nuclear matter (nuclear incompressibility $K_{\infty}=245 \mathrm{MeV}$, and nucleon effective mass $m^{*} / m=0.675$ ). For details, the reader should consult Ref. [42].

The ground state properties of ${ }^{208} \mathrm{~Pb}$ are calculated in coordinate space using box boundary conditions. The radius of the box is taken to be $20 \mathrm{fm}$ : the same box is used to calculate discrete states at positive energy that are associated with the continuum part of the spectrum. A cutoff energy of $60 \mathrm{MeV}$ (in the single-particle energy) is adopted for the RPA calculations. With this energy cutoff, we have checked that the non energy weighted sum rules for both AGDR and IAS are satisfied at the level of about $99.97 \%$ for all Skyrme functionals used in the present study.

In Fig. 2, we show the response functions corresponding to the IAS and AGDR operators obtained for ${ }^{208} \mathrm{~Pb}$ by using the SAMi-J Skyrme functionals: the RPA results have been smeared out by using Lorentzian functions. As we can see, the IAS peak energy has small fluctuations as it varies between 17.7 MeV and 18.8 MeV for the different SAMi-J parameter sets. As for the AGDR case, the peak energies vary between 26.4 MeV and 28.2 MeV by using the different SAMi-J parameter sets. Experimentally, the mean AGDR energy has been extracted from the response function in the energy interval 5-15 MeV above the IAS energy. To compare our results with the experimental findings, we shall use the same energy range to calculate the mean energy from the AGDR response.

In Fig. 3 we display the excitation energy of the AGDR and IAS as a function of the nucleon effective mass $m^{*} / m$, calculated with the SAMi-J and SAMi-m Skyrme functionals. We remind that the main difference between the SAMi-m and 

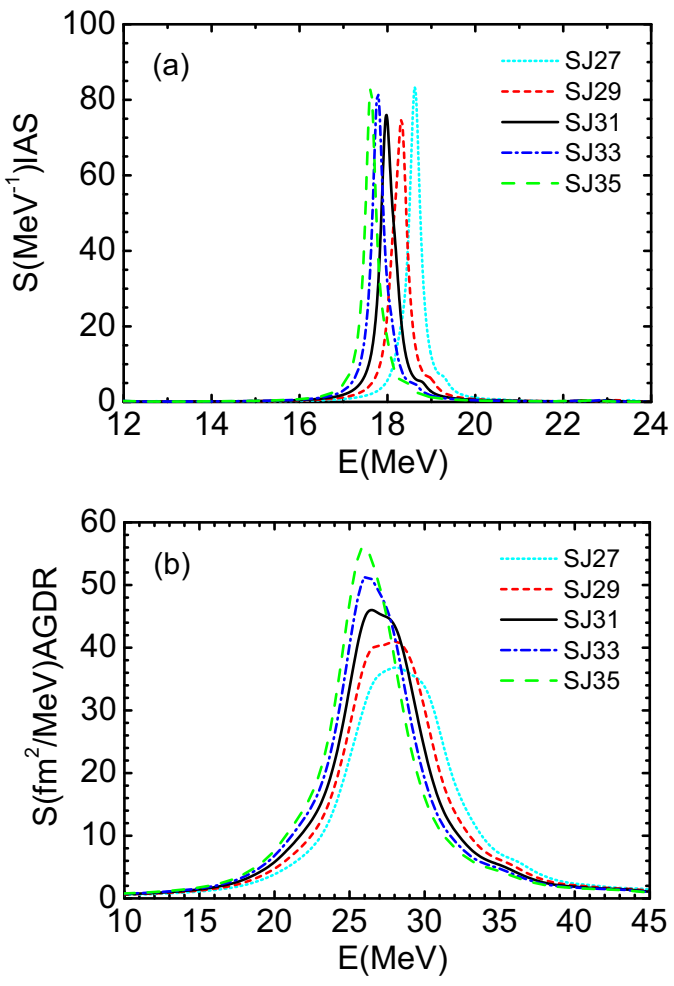

FIG. 2. (Color online) The (a) IAS and (b) AGDR response functions calculated by using the SAMi-J Skyrme energy density functionals. The discrete RPA peaks have been smeared out by using Lorentzian functions with (a) $300 \mathrm{keV}$ and (b) $3 \mathrm{MeV}$ width.

SAMi-J functionals is that in the former case the nucleon effective mass varies (in steps of 0.05) when fitting the parameters while $K_{\infty}, J$, and $L$ are kept constant (as above, we refer to [42] for details). The red squares in the panels

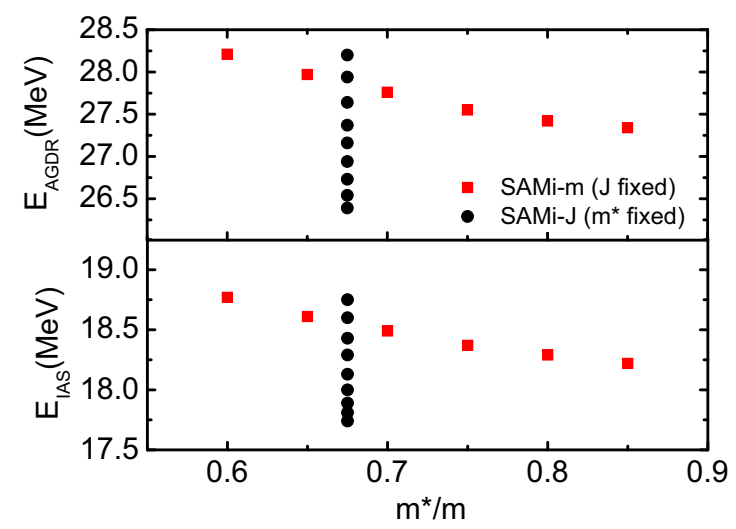

FIG. 3. (Color online) Excitation energies of the IAS and AGDR in ${ }^{208} \mathrm{~Pb}$ as a function of effective mass and symmetry energy at saturation density. The filled boxes are the results of the SAMi$\mathrm{m}$ family, while the filled circles are those of the SAMi-J family. The top circle in the line of circles of each window corresponds to the lowest $J$ value $(J=27 \mathrm{MeV})$ and by going down one increases the $J$ value in steps of $1 \mathrm{MeV}$. The lowest circle then corresponds to the maximum value, $J=35 \mathrm{MeV}$.

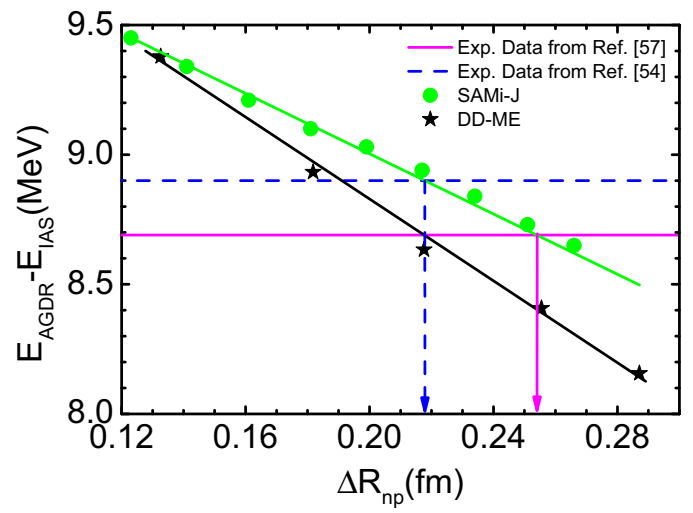

FIG. 4. (Color online) The energy difference $E_{\mathrm{AGDR}}-E_{\mathrm{IAS}}$ of AGDR and IAS as a function of neutron skin thickness, obtained by using the SAMi-J family of Skyrme functionals consistently. The calculated values are presented as solid circles. Two different experimental data [54,57] are also shown as solid (magenta) and dashed (blue) lines, respectively. The arrows indicate the neutron skin constrained by these experimental data. We also display results obtained with the covariant DD-ME Lagrangians of Refs. [52,54]. Interestingly, such models predict the same kind of correlation although with a different slope.

correspond to results from SAMi-m. The results obtained from SAMi-J (black circles) are displayed in such a way that the bottom (top) point corresponds to the highest (lowest) value of $J$. The conclusion from these panels is that the excitation energy of the AGDR is sensitive to the symmetry energy at saturation density while, as expected, the variation of the AGDR excitation energy within the sets of the family SAMi-m is small. In the case of the IAS, the excitation energy is neither sensitive to the symmetry energy nor to the effective mass.

The calculated energy differences $E_{\mathrm{AGDR}}-E_{\mathrm{IAS}}$ between AGDR and IAS, obtained by employing the SAMi-J Skyrme functionals, are displayed as a function of the corresponding neutron skin thickness in Fig. 4: in particular, the solid circles correspond to the sets SAMi-J27 to SAMi-J35, from left to right. As we mentioned above, for the excitation energy of the AGDR we take the centroid of the theoretical strength distribution, calculated in the energy interval from 5 to $15 \mathrm{MeV}$ above the IAS energy. The results show that the energy differences $E_{\mathrm{AGDR}}-E_{\mathrm{IAS}}$ between AGDR and IAS decrease with increasing values of the neutron skin thickness, and a strong linear correlation exists; this is quite well justified by the model that has been developed in Sec. III.

For the reader's convenience, we also display some results obtained using the covariant DD-ME Lagrangians of Refs. [52,54]. These obey the same kind of scaling as we have predicted with the model of Sec. III, and yet the slope is different. This illustrates, to a quite large extent, the model dependence of such kind of analysis. The two classes of functionals, SAMi and DD-ME, are recent and yet based on different theoretical frameworks (nonrelativistic and relativistic, respectively) and fitting protocols. These different fitting strategies (notably, the choices to attempt reproducing the neutron equation of state from $a b$ initio approaches and the spin-isospin Landau parameters, that are made in the case 
TABLE II. The values of the neutron skin thickness of ${ }^{208} \mathrm{~Pb}$ obtained in the present work are compared to other values extracted by means of different experimental methods.

\begin{tabular}{lccc}
\hline \hline Method & Ref. & Date & $\Delta R_{n p}(\mathrm{fm})$ \\
\hline antiproton absorption & {$[31]$} & 2001 & $0.180 \pm 0.030$ \\
$\left(\alpha, \alpha^{\prime}\right)$ IVGDR & {$[69]$} & 2004 & $0.120 \pm 0.070$ \\
PDR & {$[43]$} & 2010 & $0.194 \pm 0.024$ \\
$\left(\vec{p}, \vec{p}^{\prime}\right)$ & {$[35]$} & 2011 & $0.156 \pm 0.025$ \\
$\alpha_{D}$ & {$[41]$} & 2012 & $0.168 \pm 0.022$ \\
parity violation & {$[29]$} & 2012 & $0.330 \pm 0.170$ \\
AGDR from Exp1 & {$[57]$} & 2013 & $0.216 \pm 0.048$ \\
AGDR from Exp2 & {$[54]$} & 2013 & $0.190 \pm 0.028$ \\
$\left(\gamma, \pi^{0}\right)$ & {$[1]$} & 2014 & $0.150 \pm 0.030$ \\
AGDR from Exp1 & present & 2015 & $0.254 \pm 0.062$ \\
AGDR from Exp2 & present & 2015 & $0.218 \pm 0.015$ \\
\hline \hline
\end{tabular}

of SAMi and not in the case of DD-ME) affect the isovector properties of these functionals as discussed in Ref. [68]. As a consequence, we might infer that the comparison of SAMi and DD-ME is likely to provide a reasonable indication about the systematic error. The impact on $J$ and $L$ will be, in due course, discussed accordingly. A more systematic analysis could be envisaged as a future study.

In Fig. 4 we also superimpose two different experimental data. In Ref. [57] (that will be denoted as Exp1 hereafter), the AGDR has been separated from other excitations by means of the multipole decomposition analysis of the ${ }^{208} \mathrm{~Pb}(\vec{p}, \vec{n})$ reaction at a bombarding energy $T_{p}=296 \mathrm{MeV}$ : the polarization transfer observables have been, in this case, quite instrumental to separate the non-spin-flip AGDR from the spinflip SDR in the multipole decomposition analysis. The energy difference between the AGDR and the IAS was determined to be $E_{\mathrm{AGDR}}-E_{\mathrm{IAS}}=8.69 \pm 0.36 \mathrm{MeV}$, where the uncertainty is claimed to include both statistical and systematic contributions. We show this datum by a solid (magenta) line in Fig. 4. The other experimental measurement has been reported in Ref. [54] (Exp2): in this case, the ${ }^{208} \mathrm{~Pb}(p, n \gamma p){ }^{207} \mathrm{~Pb}$ reaction at a beam energy of $30 \mathrm{MeV}$ has been used to excite the AGDR and to measure its $\gamma$ decay to the isobaric analog state, in coincidence with proton decay of the IAS. The energy difference $E_{\mathrm{AGDR}}-E_{\mathrm{IAS}}$ between the AGDR and the IAS was determined to be $E_{\mathrm{AGDR}}-E_{\mathrm{IAS}}=8.90 \pm 0.09 \mathrm{MeV}$. We show this result by means of a dashed (blue) line in Fig. 4. Given the error bars, the two works provide consistent results.

By comparing the experimental data for the energy difference of AGDR and IAS with our correlation line, we find that the value of the neutron skin thickness of ${ }^{208} \mathrm{~Pb}$ is $\Delta R_{n p}=$ $0.254 \pm 0.062 \mathrm{fm}$ using Exp1, and $\Delta R_{n p}=0.218 \pm 0.015 \mathrm{fm}$ using Exp2, respectively: these values are indicated by arrows in Fig. 4 and reported in Table II. The weighted average of these two results would give $0.236 \pm 0.018 \mathrm{fm}$ for $\Delta R_{n p}$. We can compare our result with previous results deduced from different experimental methods. Although on the high side, our result from Exp2 is compatible with several other estimates. In Refs. [54,57], the authors also compared their experimental data with the values of $E_{\mathrm{AGDR}}-E_{\mathrm{IAS}}$ of AGDR
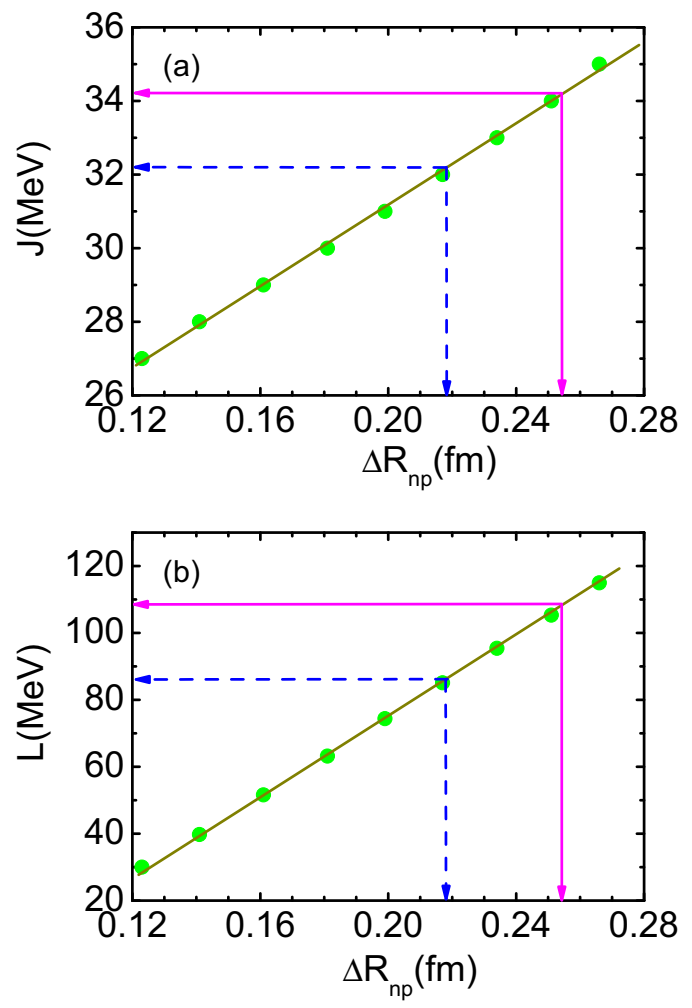

FIG. 5. (Color online) (a) and (b) show the correlations between the neutron skin thickness and either the symmetry energy $J$ at saturation density or the corresponding slope parameter $L$, respectively. The constraints provided by the experimental data have been already shown in Fig. 4.

and IAS obtained by using a fully self-consistent protonneutron relativistic RPA with a family of density-dependent meson-exchange interactions (DD-ME) [70]. Including the uncertainty both from experimental and theoretical sides (clearly, systematic errors could not be fully addressed), finally they found the value of the neutron skin thickness of ${ }^{208} \mathrm{~Pb}$ to be $\Delta R_{n p}=0.216 \pm 0.046 \pm 0.015 \mathrm{fm}$ for Exp1, and $\Delta R_{n p}=$ $0.190 \pm 0.028 \mathrm{fm}$ for Exp2, respectively [54,57]. Within such error bars, the latter results overlap with our results obtained using nonrelativistic Skyrme energy density functionals.

We have also extracted the symmetry energy $J$ and its slope parameter $L$ at saturation density by using the neutron skin thickness presently obtained. The results are shown in Fig. 5. The value for symmetry energy $J$ is extracted to be $J=$ $34.2 \pm 3.5 \mathrm{MeV}$ or $J=32.2 \pm 0.9 \mathrm{MeV}$, using Exp1 or Exp2, and the value for the slope parameter $L$ of symmetry energy at saturation density is $L=108.5 \pm 35.8 \mathrm{MeV}$ or $L=86.1 \pm$ 9.1 MeV, using Exp1 or Exp2. The weighted average of these results is $J=33.2 \pm 1.0 \mathrm{MeV}$ and $L=97.3 \pm 11.2 \mathrm{MeV}$. Of course, by making the weighted average one reduces the error bars, and this may hide even further systematic differences between the experiments and/or model dependences. As we mentioned in the abstract, and discussed above in this section, the reported errors correspond to a lower-limit estimate of the systematic plus experimental uncertainties. 


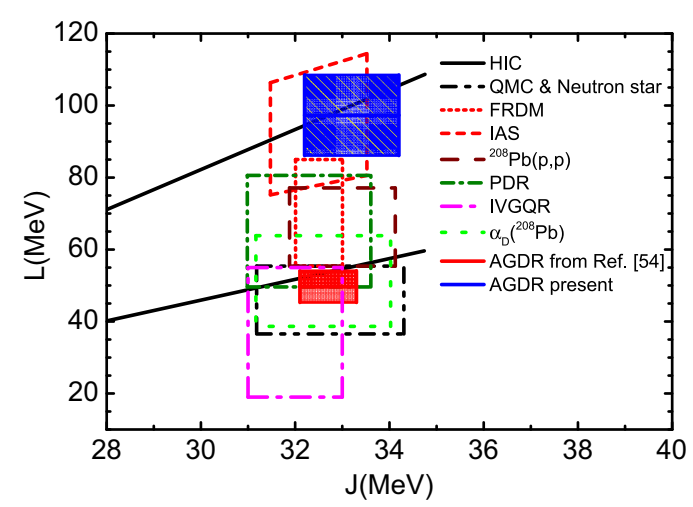

FIG. 6. (Color online) The values of the slope parameter $L$ and symmetry energy $J$ at saturation density extracted in the current work are compared with the values extracted from other experimental data with several different methods.

In Fig. 6, the extracted values of $J$ and $L$ (actually, only the weighted average in keeping with the format of the figure) by the present analysis are shown together with those obtained with other methods. These include: quantum Monte Carlo (QMC) simulations of neutron stars [71], analysis of the nuclear binding energies (by FRDM) [72], energies of isobaric analog states (IAS) [73], proton elastic scattering $\left[{ }^{208} \mathrm{~Pb}(p, p)\right]$ [30], pygmy dipole resonances (PDR) [43], total dipole polarizability [40], and excitation energy of the isovector giant quadrupole resonance [42]. We should note that the presently extracted value of $J$ is consistent with the other values in Fig. 6, with small variations. On the other hand, the present value of $L$, although similar to those from the IAS analysis and binding energies from FRDM, is somewhat larger than the average value of all other deductions.

In Ref. [54], the values of $L$ and $J$ were extracted by using the same experimental energy difference of AGDR and IAS that we have used (Exp2). They have obtained $J=32.7 \pm$ $0.6 \mathrm{MeV}$ and $L=49.7 \pm 4.4 \mathrm{MeV}$, as shown in Fig. 6 by the red shaded area. While our result for $J$ is fully consistent with the one obtained in Ref. [54], the present (central) value of $L$ is significantly larger than that of Ref. [54]. This may be due to the different energy density functionals used in the present analysis and in Ref. [54], where the RMF Lagrangians of DD-ME type were adopted. However, as discussed in the previous section, a clear explanation of this difference is a point that remains for future study.

We should also notice that in the previous studies devoted to the extraction of $L$ and $J$ from giant resonances, we have found values of $L$ like $64.8 \pm 15.7 \mathrm{MeV}$ from PDR and $37 \pm 18 \mathrm{MeV}$ from IVGQR as reviewed in [50]. These values are smaller than the present values: the present value is consistent with one of the previous estimates but not with both of them. It remains as an open question to be fixed, to which extent such discrepancies are related to the different energy functionals used for the different estimates, to experimental errors, or to some more basic flaw. It should be stressed, on the other hand, that the presently estimated value of $J$ is consistent with other extractions from giant resonance data.

\section{SUMMARY AND PERSPECTIVE}

In this work, we have studied the correlation of the neutron skin thickness and the energy difference $E_{\mathrm{AGDR}}-E_{\mathrm{IAS}}$ of AGDR and IAS in ${ }^{208} \mathrm{~Pb}$, by using a family of effective Skyrme energy density functionals, named SAMi-J (SAMi-m), that are characterized by different values of symmetry energy $J$ (effective mass $m^{*}$ ). The calculations have been done within a fully self-consistent Skyrme HF plus charge-exchange RPA framework. We find a strong linear correlation of the energy difference $E_{\mathrm{AGDR}}-E_{\mathrm{IAS}}$ with the neutron skin thickness $\Delta R_{n p}$ in ${ }^{208} \mathrm{~Pb}$. An analytic model has been developed to explain the dependence of the excitation energy of AGDR on the neutron skin thickness, in which it becomes apparent that such excitation energy decreases when the neutron skin thickness increases. We also confirmed that the symmetry energy $J$ and the slope parameter $L$ have linear correlations with the neutron skin thickness within the employed Skyrme SAMi-J models.

Accordingly, we have extracted the neutron skin thickness in ${ }^{208} \mathrm{~Pb}$ as $\Delta R_{n p}=0.254 \pm 0.062 \mathrm{fm}$ using Exp1, and $\Delta R_{n p}=0.218 \pm 0.015 \mathrm{fm}$ using Exp2, respectively. We have also constrained the symmetry energy $(J=34.2 \pm 3.5 \mathrm{MeV}$ or $J=32.2 \pm 0.9 \mathrm{MeV}$, from either Exp1 or Exp2) and its slope parameter $(L=108.5 \pm 35.8 \mathrm{MeV}$ or $L=86.1 \pm 9.1 \mathrm{MeV}$ from Exp1 or Exp2) at saturation density by using the value of the neutron skin. The averaged values from the two experiments are $\Delta R_{n p}=0.236 \pm 0.018$ fm, $J=33.2 \pm 1.0 \mathrm{MeV}$ and $L=97.3 \pm 11.2 \mathrm{MeV}$. Good agreement is obtained in comparing with our new results for the neutron skin thickness and the symmetry energy $J$ with the values extracted with many different experimental methods within the error bars. On the other hand, the presently extracted $L$ value is somewhat larger than the previously obtained values. The reported errors in our theoretical analysis correspond to a lower-limit estimate of the systematic plus experimental uncertainties. In fact, our discussion of Fig. 4 has shown that improvements, both in our understanding of the properties of the charge-exchange modes and in assessing model dependences of the extraction of symmetry energy parameters, would be of paramount importance.

The use of the $(p, n)$ reaction to study the AGDR can be extended to unstable nuclei due to the progress made in the development of new experimental techniques involving radioactive beams in inverse kinematics [74,75]. Further experimental efforts on the AGDR in other mass regions and/or in long isotopic chains are desirable to increase the predictive power of current energy density functionals and to reduce the model dependence that one deals with when extracting nuclear matter properties. This may eventually allow us to better constrain the equation of state of asymmetric nuclear matter, a landmark for nuclear physics and nuclear astrophysics.

To pursue our research, whenever data on open-shell nuclei become available, we need of course to include pairing correlations. We already dispose of fully self-consistent quasiparticle RPA (QRPA) implementations on top of either HF-Bardeen-Cooper-Schrieffer or Hartree-Fock-Bogoliubov approximations for the ground state: these approaches are 
discussed in Refs. [76,77], respectively. This can allow extensions of our study, with the only limitation related to the assumption of spherical symmetry for the nuclei under study.

\section{ACKNOWLEDGMENTS}

This work is supported by the National Natural Science Foundation of China under Grant Nos. 11175216, 11575060, and 11435014, and the Fundamental Research Funds for the Central Universities (JB2014241).
[1] C. M. Tarbert et al., Phys. Rev. Lett. 112, 242502 (2014).

[2] T. Suzuki et al., Phys. Rev. Lett. 75, 3241 (1995).

[3] A. Krasznahorkay et al., Phys. Rev. Lett. 66, 1287 (1991).

[4] B. K. Agrawal, J. N. De, and S. K. Samaddar, Phys. Rev. Lett. 109, 262501 (2012).

[5] X. Roca-Maza, M. Centelles, X. Viñas, and M. Warda, Phys. Rev. Lett. 106, 252501 (2011).

[6] M. Centelles, X. Roca-Maza, X. Viñas, and M. Warda, Phys. Rev. Lett. 102, 122502 (2009).

[7] D. Vretenar, N. Paar, T. Nikšić, and P. Ring, Phys. Rev. Lett. 91, 262502 (2003).

[8] R. J. Furnstahl, Nucl. Phys. A 706, 85 (2002).

[9] C. J. Horowitz and J. Piekarewicz, Phys. Rev. Lett. 86, 5647 (2001).

[10] B. A. Brown, Phys. Rev. Lett. 85, 5296 (2000).

[11] A. E. L. Dieperink, Y. Dewulf, D. Van Neck, M. Waroquier, and V. Rodin, Phys. Rev. C 68, 064307 (2003)

[12] M. B. Tsang et al., Phys. Rev. C 86, 015803 (2012).

[13] P. Danielewicz, R. Lacey, and W. G. Lynch, Science 298, 1592 (2002).

[14] L. W. Chen, C. M. Ko, and B. A. Li, Phys. Rev. C 72, 064309 (2005).

[15] L. W. Chen, C. M. Ko, B. A. Li, and J. Xu, Phys. Rev. C 82, $024321(2010)$.

[16] C. Xu, B.-A. Li, and L.-W. Chen, Phys. Rev. C 82, 054607 (2010).

[17] G.-C. Yong, B.-A. Li, L.-W. Chen, and W. Zuo, Phys. Rev. C 73, 034603 (2006).

[18] Z. Xiao, B.-A. Li, L.-W. Chen, G.-C. Yong, and M. Zhang, Phys. Rev. Lett. 102, 062502 (2009).

[19] Z. Q. Feng and G. M. Jin, Phys. Lett. B 683, 140 (2010).

[20] Y. Gao, L. Zhang, W. Zuo, J.-Q. Li, Phys. Rev. C 86, 034611 (2012).

[21] J. Dong, W. Zuo, and W. Scheid, Phys. Rev. Lett. 107, 012501 (2011).

[22] B. A. Li, Nucl. Phys. A 708, 365 (2002).

[23] J. Rizzo et al., Nucl. Phys. A 806, 79 (2008).

[24] W. G. Newton et al., Eur. Phys. J. A 50, 41 (2014).

[25] J. M. Lattimer, Nucl. Phys. A 928, 276 (2014).

[26] S. Gandolfi, J. Carlson, and S. Reddy, Phys. Rev. C 85, 032801 (2012).

[27] M. Kutschera, Phys. Lett. B 340, 1 (1994).

[28] M. K. Gaidarov, A. N. Antonov, P. Sarriguren, and E. Moya de Guerra, Phys. Rev. C 84, 034316 (2011).

[29] S. Abrahamyan et al., Phys. Rev. Lett. 108, 112502 (2012).

[30] J. Zenihiro et al., Phys. Rev. C 82, 044611 (2010).

[31] A. Trzcińska, J. Jastrzebski, P. Lubiński, F. J. Hartmann, R. Schmidt, T. von Egidy, and B. Kłos, Phys. Rev. Lett. 87, 082501 (2001).

[32] B. Kłos et al., Phys. Rev. C 76, 014311 (2007).

[33] P. Adrich et al., Phys. Rev. Lett. 95, 132501 (2005).
[34] O. Wieland et al., Phys. Rev. Lett. 102, 092502 (2009).

[35] A. Tamii et al., Phys. Rev. Lett. 107, 062502 (2011).

[36] D. M. Rossi et al., Phys. Rev. Lett. 111, 242503 (2013).

[37] A. Klimkiewicz et al., Phys. Rev. C 76, 051603(R) (2007).

[38] L. Trippa, G. Colò, and E. Vigezzi, Phys. Rev. C 77, 061304(R) (2008).

[39] L. G. Cao and Z. Y. Ma, Chin. Phys. Lett. 25, 1625 (2008).

[40] X. Roca-Maza, M. Brenna, G. Colò, M. Centelles, X. Viñas, B. K. Agrawal, N. Paar, D. Vretenar, and J. Piekarewicz, Phys. Rev. C 88, 024316 (2013).

[41] J. Piekarewicz et al., Phys. Rev. C 85, 041302(R) (2012).

[42] X. Roca-Maza, M. Brenna, B. K. Agrawal, P. F. Bortignon, G. Colò, L. G. Cao, N. Paar, and D. Vretenar, Phys. Rev. C 87, 034301 (2013).

[43] A. Carbone, G. Colò, A. Bracco, L. G. Cao, P. F. Bortignon, F. Camera, and O. Wieland, Phys. Rev. C 81, 041301(R) (2010).

[44] J. Piekarewicz, Phys. Rev. C 83, 034319 (2011).

[45] Z. Zhang and L. W. Chen, Phys. Rev. C 90, 064317 (2014).

[46] A. Krasznahorkay et al., Phys. Rev. Lett. 82, 3216 (1999).

[47] K. Yako et al., Phys. Lett. B 615, 193 (2005).

[48] K. Yako, H. Sagawa, and H. Sakai, Phys. Rev. C 74, 051303(R) (2006).

[49] H. Sagawa, S. Yoshida, X.-R. Zhou, K. Yako, and H. Sakai, Phys. Rev. C 76, 024301 (2007).

[50] G. Colò, U. Garg, and H. Sagawa, Eur. Phys. J. A 50, 26 (2014).

[51] Bui Minh Loc, Dao T. Khoa, and R. G. T. Zegers, Phys. Rev. C 89, 024317 (2014).

[52] A. Krasznahorkay, N. Paar, D. Vretenar, and M. N. Harakeh, Phys. Scr. T 154, 014018 (2013).

[53] A. Krasznahorkay, N. Paar, D. Vretenar, and M. N. Harakeh, Phys. Lett. B 720, 428 (2013).

[54] A. Krasznahorkay et al., arXiv:1311.1456.

[55] W. A. Sterrenburg, S. M. Austin, R. P. DeVito, and A. Galonsky, Phys. Rev. Lett. 45, 1839 (1980).

[56] F. Krmpotic, K. Nakayama, and A. Pio Galeao, Nucl. Phys. A 399, 478 (1983).

[57] J. Yasuda et al., Prog. Theor. Exp. Phys. (2013) 063 D02.

[58] D. Vautherin and D. M. Brink, Phys. Rev. C 5, 626 (1972).

[59] E. Chabanat et al., Nucl. Phys. A 635, 231 (1998).

[60] P. Ring and P. Schuck, The Nuclear Many-Body Problem (Springer-Verlag, New York, 1980).

[61] D. J. Rowe, Nuclear Collective Motion: Models and Theory (Methuen, London, 1970).

[62] G. Colò, N. Van Giai, P. F. Bortignon, and R. A. Broglia, Phys. Rev. C 50, 1496 (1994).

[63] G. Colò, L. G. Cao, N. V. Giai, and L. Capelli, Comp. Phys. Commun. 184, 142 (2013).

[64] A. Bohr and B. R. Mottelson, Nuclear Structure, Vols. I and II (W. A. Benjamin, Inc., Reading, MA, 1975). 
[65] M. Harakeh and A. van der Woude, Giant Resonances. Fundamental High-Frequency Modes of Nuclear Excitations (Clarendon Press, Oxford, 2011).

[66] N. Auerbach, A. Klein, and Nguyen van Giai, Phys. Lett. B 106, 347 (1981).

[67] M. Centelles, X. Roca-Maza, X. Viñas, and M. Warda, Phys. Rev. C 82, 054314 (2010).

[68] X. Roca-Maza, N. Paar, and G. Colò, J. Phys. G: Nucl. Part. Phys. 42, 034033 (2015).

[69] A. Krasznahorkay et al., Nucl. Phys. A 731, 224 (2004).

[70] N. Paar, P. Ring, T. Nikšić, and D. Vretenar, Phys. Rev. C 67, 034312 (2003); D. Vretenar, T. Nikšić, and P. Ring, ibid. 68, 024310 (2003); N. Paar, T. Nikšić, D. Vretenar, and P. Ring, ibid. 69, 054303 (2004).

[71] A. W. Steiner and S. Gandolfi, Phys. Rev. Lett. 108, 081102 (2012).

[72] P. Möller, W. D. Myers, H. Sagawa, and S. Yoshida, Phys. Rev. Lett. 108, 052501 (2012).

[73] P. Danielewicz and J. Lee, Nucl. Phys. A 818, 36 (2009).

[74] M. Sasano et al., Phys. Rev. Lett. 107, 202501 (2011).

[75] M. Sasano et al., Phys. Rev. C 86, 034324 (2012).

[76] S. Fracasso and G. Colò, Phys. Rev. C 72, 064310 (2005).

[77] C. L. Bai, H. Sagawa, G. Colò, Y. Fujita, H. Q. Zhang, X. Z. Zhang, and F. R. Xu, Phys. Rev. C 90, 054335 (2014). 